\title{
Strangeness Vector and Axial-Vector Form Factors of the Nucleon
}

\author{
Stephen Pate ${ }^{1, a}$ and Dennis Trujillo ${ }^{1}$ \\ ${ }^{1}$ Physics Department, New Mexico State University, Las Cruces NM 88003, USA
}

\begin{abstract}
A revised global fit of electroweak $e p$ and $v p$ elastic scattering data has been performed, with the goal of determining the strange quark contribution to the vector and axial-vector form factors of the nucleon in the momentum-transfer range $0<Q^{2}<1 \mathrm{GeV}^{2}$. The two vector (electric and magnetic) form factors $G_{E}^{s}\left(Q^{2}\right)$ and $G_{M}^{s}\left(Q^{2}\right)$ are strongly constrained by ep elastic scattering data, while the major source of information on the axial-vector form factor $G_{A}^{s}\left(Q^{2}\right)$ is $v p$ scattering data. Combining the two kinds of data into a single global fit makes possible additional precision in the determination of these form factors, and provides a unique way to determine the strange quark contribution to the nucleon spin, $\Delta S$, independently of leptonic deep-inelastic scattering. The fit makes use of data from the BNL-E734, SAMPLE, HAPPEx, G0, and PVA4 experiments; we will also compare the result of the fit with recent data from MiniBooNE, and anticipate how this fit can be improved when new data from MicroBooNE become available.
\end{abstract}

\section{Overview}

The strange quark contribution to the elastic form factors of the nucleon has been the subject of intense experimental scrutiny for several decades. Experiments involving elastic scattering of neutrinos and anti-neutrinos (BNL E734 [1]), and electrons (SAMPLE [2], HAPPEx [3-7], G0 [8, 9], and PVA4 [10-12]) from nucleons and nuclei have explored the strange quark presence in the nucleon by exploiting the electromagnetic and weak interactions in a variety of ways. These special data permit a simultaneous determination [13] of the strange quark contribution to the electric $\left(G_{E}^{s}\left(Q^{2}\right)\right)$, magnetic $\left(G_{M}^{s}\left(Q^{2}\right)\right)$, and axial $\left(G_{A}^{s}\left(Q^{2}\right)\right)$ form factors of the nucleon, which respectively allow us to understand how the strange quark contributes to the distribution of charge, current, and spin inside the nucleon. A global fit of these data has been performed, which sets strong limits on the size and $Q^{2}$-dependence of $G_{E}^{s}\left(Q^{2}\right)$ and $G_{M}^{s}\left(Q^{2}\right)$, and points out the need for additional neutrino scattering data at low $Q^{2}$ to complete a measurement of $G_{A}^{s}\left(Q^{2}\right)$.

\section{Strangeness Form Factors}

Since the strange and anti-strange quarks have opposite electric charges, then they contribute to the strangeness electric form factor $G_{E}^{s}$ with opposite sign; therefore, if the $s$ and $\bar{s}$ distributions in the

\footnotetext{
a e-mail: pate@nmsu.edu
} 
nucleon are the same, then $G_{E}^{s}$ will be zero. Likewise, $s$ and $\bar{s}$ contribute oppositely to the strangeness magnetic form factor $G_{M}^{s}$, and so any similarity between the $s$ and $\bar{s}$ distributions will drive $G_{M}^{s}$ to small values. However, $s$ and $\bar{s}$ have the same axial coupling, so if the $s$ and $\bar{s}$ distributions are similar then a non-zero strangeness axial form factor $G_{A}^{s}$ can arise.

\section{Experiments}

To access the strangeness vector form factors of the nucleon, there has been a series of measurements of parity-violating asymmetries in elastic electron-nucleon scattering [14], in which the electron is longitudinally polarized and the asymmetry in the cross section arises from the parity-violating nature of $Z$-exchange. In forward scattering, the PV asymmetries are most sensitive to the electric form factor, whereas in backward scattering there is a significant contribution also from the magnetic and axial form factors. In neutrino-nucleon scattering, on the other hand, the most significant contribution comes from the axial form factor. To date, the only useful measurement of the neutrino-proton elastic scattering cross section is from BNL Experiment 734 [1]. Combining these two kinds of experiments together, allows the simultaneous determination of the strange quark contribution to the vector and axial form factors [13].

\section{Determination of the Strangeness Form Factors at Specific Values of $Q^{2}$}

Quite a number of analyses have been done, in which only the PV electron-scattering data are used and so only the strangeness vector form factors $G_{E}^{s}$ and $G_{M}^{s}$ are extracted. Using the techniques developed in [16], it was possible to determine also the strangeness axial form factor at a number of points where the HAPPEx and G0 experiments overlapped with the E734 data. All of these determinations are shown in Figure 1. It is seen that both $G_{E}^{s}$ and $G_{M}^{s}$ are consistent with zero across the range $0<Q^{2}<1 \mathrm{GeV}^{2}$, whereas in the axial form factor $G_{A}^{s}$ there is a signal for a non-zero value as we approach $Q^{2}=0$.

\section{Global Fit of the Strangeness Form Factors}

Taken together, there are 48 data points from E734, SAMPLE, HAPPEx, G0 and PVA4. Not all of these are used in the determinations shown in FIgure 1. To make use of all this data, it necessary to assume functional forms for the strangeness form factors and perform a global fit.

Based on the results of the determinations at specific values of $Q^{2}$, we have fit the form factors $G_{E}^{s}, G_{M}^{s}$, and $G_{A}^{s}$ in the range $0<Q^{2}<1 \mathrm{GeV}^{2}$ with this simple set of functional forms:

$$
G_{E}^{s}=\rho_{s} \tau \quad G_{M}^{s}=\mu_{s} \quad G_{A}^{s}=\frac{\Delta S+S_{A} Q^{2}}{\left(1+Q^{2} / \Lambda_{A}^{2}\right)^{2}}
$$

where $\tau=Q^{2} / 4 M_{N}^{2},\left.\rho_{s} \equiv\left(d G_{E}^{s} / d \tau\right)\right|_{\tau=0}$ is the strangeness radius, $\mu_{s}$ is the strangeness magnetic moment, $\Delta S$ is the strange quark contribution to the nucleon spin, and $\Lambda_{A}$ and $S_{A}$ determine the $Q^{2}$ dependence of $G_{A}^{s}$.

These are the simplest functions that are consistent with the determinations shown in Figure 1. Since $G_{E}^{s}$ must be 0 at $Q^{2}=0$, then the lowest order term in $G_{E}^{s}$ must be linear in $Q^{2}$. The lowest order term in $G_{M}^{s}$ is a constant, since $G_{M}^{s}$ need not be 0 at $Q^{2}=0$. For $G_{A}^{s}$, a more complex form is required.

The (preliminary) best values for the five fit parameters are given in Table 1 . We see that the parameters representing the vector form factors $\left(\rho_{s}\right.$ and $\left.\mu_{s}\right)$ are consistent with zero, as expected. 

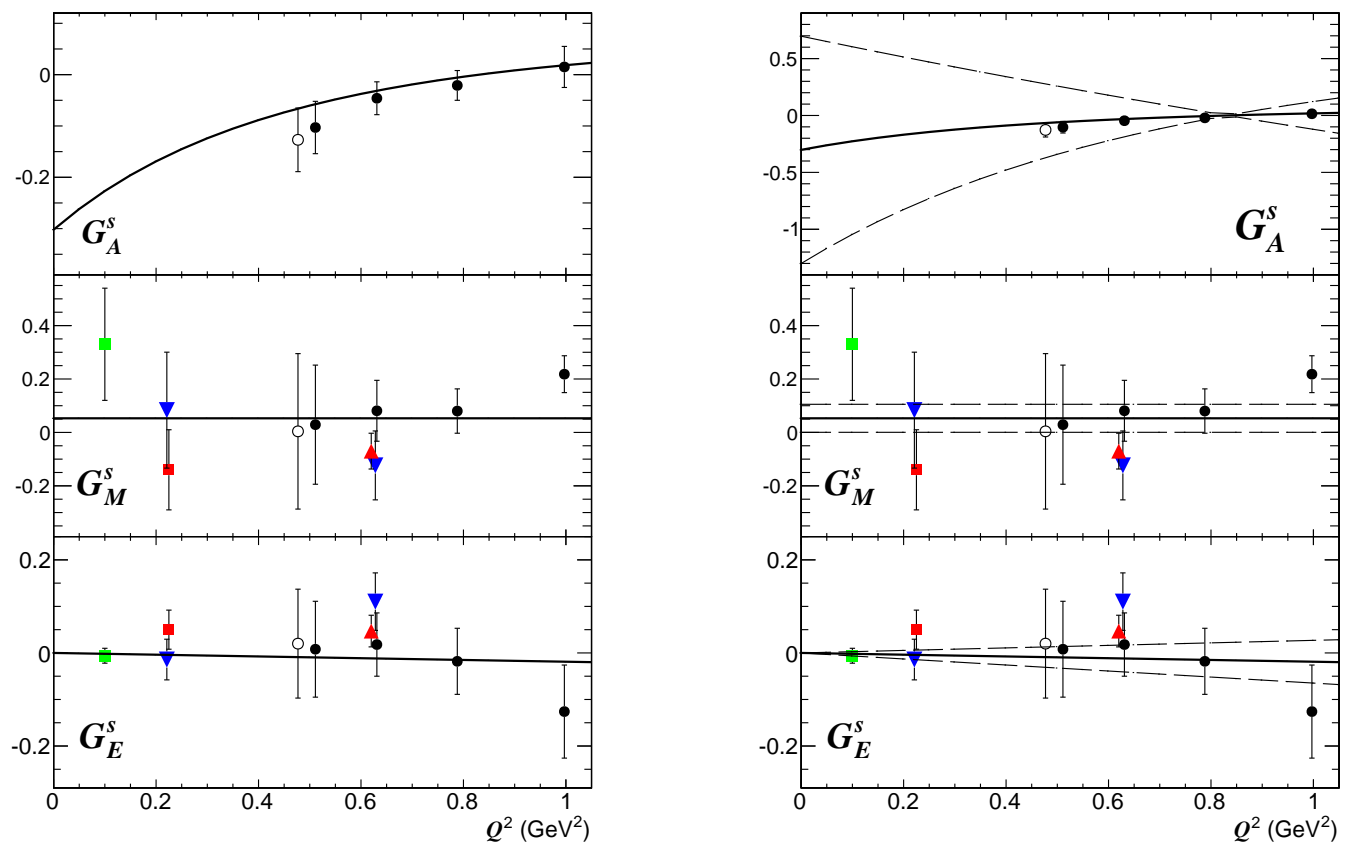

Figure 1. Results of the determination of $G_{E}^{s}, G_{M}^{s}$, and $G_{A}^{s}$ at individual values of $Q^{2}$, and also from our global fit. The separate determinations were done by Liu et al. [15] (green squares at $0.1 \mathrm{GeV}^{2}$ ), Androić et al. [9] (blue inverted triangles), Baunack et al. [12] (red squares at $0.23 \mathrm{GeV}^{2}$ ), Ahmed et al. [7] (red triangles at $0.62 \mathrm{GeV}^{2}$ ), and Pate et al. [16] (open and closed circles). The preliminary results of our global fit (see text) are shown by the solid line; the $70 \%$ confidence level limit curves for the fit are shown as the dashed line in the right-hand panel. The vertical scale for $G_{A}^{s}$ in the right-hand panel has been adjusted to accommodate the limit curves of the fit.

Strong limits are placed on the contribution of the strange quarks to the vector form factors throughout this $Q^{2}$ range. On the other hand, $\Delta S$ is also consistent with 0 but the uncertainty is very large because there are no $v p$ or $\bar{v} p$ elastic data at sufficiently low $Q^{2}$ to constrain it. As a result the uncertainties in the global fit to $G_{A}^{s}$ are very much larger than the uncertainties in the separate determinations of $G_{A}^{s}$ in Figure 1. We cannot determine $\Delta S$ in this method until additional neutrino scattering data are obtained at low $Q^{2}$.

Table 1. Preliminary results for our 5-parameter fit to the 48 elastic neutrino- and PV electron-scattering data points from BNL E734, HAPPEx, SAMPLE, G0, and PVA4.

\begin{tabular}{cc}
\hline Parameter & Fit value \\
\hline$\rho_{S}$ & $-0.071 \pm 0.096$ \\
$\mu_{s}$ & $0.053 \pm 0.029$ \\
$\Delta S$ & $-0.30 \pm 0.42$ \\
$\Lambda_{A}$ & $1.1 \pm 1.1$ \\
$S_{A}$ & $0.36 \pm 0.50$ \\
\hline
\end{tabular}




\section{Measurement of neutrino-proton elastic scattering at MicroBooNE}

MicroBooNE (http://www-microboone.fnal.gov/) is a new neutrino-scattering experiment under construction at Fermilab, consisting of a 170-ton liquid-argon time projection chamber to be placed in the path of a beam of approximately $1 \mathrm{GeV}$ neutrinos. This detector is ideal for observing neutrino-proton elastic scattering events, as low-energy protons from these events can travel several centimeters in liquid argon; a measurement down to $Q^{2}=0.08 \mathrm{GeV}^{2}$ is possible. A determination of $G_{A}^{s}$ down to such a low value of $Q^{2}$ would permit a determination of $\Delta S$. To estimate the level of uncertainty of such a measurement, a simulation of $2 \times 10^{20}$ protons-on-target was performed (about one running year), using reasonable event selection cuts. ${ }^{1}$ These simulated MicroBooNE cross section measurements were then fed back into our global fit program, and we observed the change in the uncertainties in the fit parameters; see the table below. It is seen that a measurement of the strangeness axial form factor

Table 2. Improvement in uncertainties in fit parameters for $G_{A}^{s}$, when simulated MicroBooNE data are included in the fit.

\begin{tabular}{ccc}
\hline Parameter & Existing Data & Including MicroBooNE \\
\hline$\Delta S$ & \pm 0.42 & \pm 0.038 \\
$\Lambda_{A}$ & \pm 1.1 & \pm 0.38 \\
$S_{A}$ & \pm 0.50 & \pm 0.071 \\
\hline
\end{tabular}

at MicroBooNE can have a dramatic effect on this analysis, and we look forward to a determination of $\Delta S$ to come from this project in the next few years.

\section{Acknowledgments}

This work was funded by the US Department of Energy, Office of Science.

\section{References}

[1] L.A. Ahrens et al., Phys. Rev. D35, 785 (1987)

[2] E.J. Beise, M.L. Pitt, D.T. Spayde, Prog. Part. Nucl. Phys. 54, 289 (2005), nucl-ex/0412054

[3] K.A. Aniol et al. (HAPPEx), Phys. Rev. C69, 065501 (2004), nucl-ex/0402004

[4] K.A. Aniol et al. (HAPPEx), Phys. Lett. B635, 275 (2006), nucl-ex/0506011

[5] K.A. Aniol et al. (HAPPEx), Phys. Rev. Lett. 96, 022003 (2006), nucl-ex/0506010

[6] A. Acha et al. (HAPPEx), Phys. Rev. Lett. 98, 032301 (2007), nucl-ex/0609002

[7] Z. Ahmed et al. (HAPPEX collaboration), Phys.Rev.Lett. 108, 102001 (2012), 1107.0913

[8] D.S. Armstrong et al. (G0), Phys. Rev. Lett. 95, 092001 (2005), nucl-ex/0506021

[9] D. Androić et al. (G0), Phys. Rev. Lett. 104, 012001 (2010), 0909. 5107

[10] F.E. Maas et al. (A4), Phys. Rev. Lett. 93, 022002 (2004), nucl-ex/0401019

[11] F.E. Maas et al. (A4), Phys. Rev. Lett. 94, 152001 (2005), nucl-ex/0412030

[12] S. Baunack et al. (A4), Phys. Rev. Lett. 102, 151803 (2009), 0903. 2733

[13] S.F. Pate, Phys. Rev. Lett. 92, 082002 (2004), hep-ex/0310052

[14] D. Armstrong, R. McKeown, Ann.Rev.Nucl.Part.Sci. 62, 337 (2012), 1207.5238

[15] J. Liu, R.D. McKeown, M.J. Ramsey-Musolf, Phys. Rev. C76, 025202 (2007), 0706.0226

[16] S.F. Pate, D.W. McKee, V. Papavassiliou, Phys. Rev. C78, 015207 (2008), 0805.2889

\footnotetext{
${ }^{1}$ Thanks to B. Fleming, J. Spitz, and V. Papavassiliou for providing this simulation.
} 